\title{
Respiratory Sinus Arrhythmia is Reduced in Adolescent Major DEPRESSIVE DISORDER
}

\author{
I. Tonhajzerova ${ }^{1}$, I. Ondrejka², M. Javorka ${ }^{1}$, P. Adamik², Z. Turianikova¹, V. Kerna², K. Javorka ${ }^{1}$, \\ A. Calkovska ${ }^{1}$ \\ ${ }^{1}$ Institute of Physiology, ${ }^{2}$ Psychiatric Clinic, Jessenius Faculty of Medicine, Comenius University, \\ Martin Faculty Hospital, Martin, Slovakia
}

\begin{abstract}
Objective: Although the emotion regulatory difficulties in patients with major depressive disorder (MDD) are predicted to associate with impaired cardiovascular autonomic regulation, the changes of cardiac vagal regulation in MDD are incompletely understood. The aim of the study was to evaluate the respiratory sinus arrhythmia (as an index of cardiac vagal regulation) using the spectral analysis in high frequency band of the heart rate variability and the indices of deep breathing test in adolescent patients with major depressive disorder.

Material and methods: Twenty-eight adolescent girls were examined - 14 patients with major depressive disorder without pharmacological treatment (average age: $16.4 \pm 0.2 \mathrm{yr}$ ) and 14 healthy probands (control group) matched for age and gender. The respiratory sinus arrhythmia was evaluated using the spectral analysis in high frequency band of the heart rate variability (HF-HRV) and the parameters of deep breathing test (I-E, I/E). In addition, mean R-R interval was calculated.

Results: The adolescent patients with MDD had significantly reduced spectral activity in the HF-HRV and lower I/E, I-E parameters compared to matched healthy subjects $(\mathrm{P}<0.05)$.

Conclusions: We conclude that the adolescent girls with MDD have reduced respiratory sinus arrhythmia indicating cardiac vagal dysregulation. Since impaired cardiac vagal regulation is associated with increased risk of cardiovascular morbidity, this finding underscores the importance of impaired autonomic neurocardiac integrity already in adolescents with major depressive disorder without pharmacological treatment.
\end{abstract}

Key words: respiratory sinus arrhythmia, heart rate variability, deep breathing, cardiac vagal regulation, major depressive disorder, adolescent age

\section{INTRODUCTION}

Respiratory-frequency rhythms are translated into changes in a discharge frequency of the sinoatrial node known as respiratory sinus arrhythmia (RSA).
RSA is mediated through physiological mechanisms by which the heart rate increases during inspiration and decreases during expiration. These mechanisms include central medullary generator, reflexes from the lungs, baroreflexes, chemoreflexes, as well as local mechanisms (stretching of the sinoatrial node etc.). RSA is mediated predominantly by fluctuations of vagal cardiac nerve efferent traffic originating in the nucleus ambiguus and therefore provides a non-invasive index of cardiac vagal regulation $[1,2]$. Moreover, the cardiac vagal modulation as reflected in RSA has been shown to be related to emotional regulation $[3,4]$.

RSA can be quantified by various methods - the spectral analysis of the heart rate variability (HRV) at respiratory-linked high frequency (HF-HRV) neuronal oscillations, or the respiratory manoeuvre - deep breathing test. Previous studies have documented correlation between HF-HRV and cerebral blood flow in certain cortical (prefrontal, insular cortex) and subcortical regions [5]. Thus, the spectral analysis in high frequency band of the heart rate variability is considered as an index of central-peripheral neural feedback and central-autonomic nervous system integration $[6,7]$.

Major depressive disorder (MDD) is being conceptualized as a disorder of emotion regulation. Despite the clear emotion regulatory difficulties characterizing depressive patients, the results related to the RSA (an index of cardiac vagal control) and depression are mixed. Some authors report no differences in respiratory sinus arrhythmia between depressed subjects and non-depressed controls [8], while others have found reduced cardiac vagal modulation in patients with MDD [9]. Thus, the effects of MDD on the cardiac autonomic regulation remain controversial. We chose to evaluate these effects in adolescent patients since the adolescence is a critical period for maturation of higher cognitive functions and emotional behaviour [10]. Thus, the adolescent subjects may be more prone to depression-induced cardiac autonomic dysregulation and more vulnerable to its long-term consequences.

We addressed the hypothesis that respiratory sinus arrhythmia is altered in the adolescent girls with MDD. Therefore, the aim of the study was to evaluate the 
respiratory sinus arrhythmia, as a non-invasive index of cardiac vagal regulation, using the spectral analysis in high frequency band of the heart rate variability and deep breathing test in adolescent patients with major depressive disorder without pharmacological intervention.

\section{MATERIAL AND METHODS}

\section{SUBJECTS}

The study was approved by the Ethics Committee of Jessenius Medical Faculty, Comenius University. All subjects and their parents were carefully instructed about the study protocol and they gave written informed consent to participate in the study prior to examination.

We have examined 28 adolescent girls - 14 patients with major depressive disorder, single episode (average age: $16.4 \pm 0.2 \mathrm{yr}$ ) and 14 healthy subjects matched for age and gender (control group). The major depressive disorder was classified according to Diagnostic and Statistical Manual of Mental Disorders, Fourth Edition, Text Revision (DSM-IV-TR) [11]. No subject had any history of cardiovascular, respiratory, endocrinological, neurological, or other disorder known to affect HRV (including alcohol or drug abuse). Additionally, the smokers were excluded from this study.

\section{PROTOCOL}

All subjects were examined under standard conditions (quiet room with minimal arousal stimuli, standard temperature) from 8:00 a.m. to $11: 30$ a.m. The subjects were instructed to lie back comfortably and not to speak or move unless necessary. The thoracic belt with ECG electrodes from telemetric device for R-R intervals recording VarCorPF6 (Dimea, Olomouc, Czech Republic) was applied after initial $15 \mathrm{~min}$. of the rest. Then, the deep breathing test was performed. After deep breathing test the subjects remained in the supine position for the continuous ECG recording.

\section{DATA ANALYSIS}

The R-R intervals time series were obtained from VarCorPF6 device (sampling frequency of $1000 \mathrm{~Hz}$ ) according to standards recommended by Task Force [12].
Spontaneous HRV: The $300 \mathrm{R}-\mathrm{R}$ intervals segments were analyzed between the first and fifth minute of the supine position. Slower oscillations and trends were eliminated using the detrending procedure of Tairvanen et al. [13] and time series were resampled (resampling frequency of $2 \mathrm{~Hz}$ ) to obtain equidistant time series using cubic spline interpolation. Subsequently, mean power spectrum of the analysed segment was computed by fast Fourier transform (using window length of 256 samples) and spectral power in the high frequency band (HF: $0.15-0.4 \mathrm{~Hz}$ ) was obtained by integration. We have focused on the high frequency spectral power of the HRV (HF-HRV) reflecting mainly respiratory sinus arrhythmia. In addition, mean R-R interval was calculated.

Deep breathing test: The subject lying in the supine position was instructed to perform four maximal inspirations and expirations in twenty seconds. The following parameters were evaluated: I-E - difference of the mean heart rate during maximal inspirations and expirations, I/E - ratio of these values. These indices describe a magnitude of the respiratory sinus arrhythmia.

Statistics: Data were expressed as means \pm SE. The non-Gaussian distribution of the spectral indices was ascertained using Lilliefors test. Therefore, the HFHRV index was logarithmically transformed for subsequent statistical analysis. A Mann-Whitney U-test was used for statistical analysis of the parameters with non-Gaussian distribution and Student's two-sample ttest was performed to assess statistical differences in variables with normal distribution. $\mathrm{P}<0.05$ was considered as statistically significant.

\section{RESULTS}

Table 1 shows the heart rate variability and deep breathing test results. The mean R-R interval was marginally significantly shorter in depression group compared to controls $(\mathrm{P}=0.053)$. The high frequency oscillations of the heart rate variability (HF-HRV) were significantly reduced in depression group compared to controls $(\mathrm{P}=0.026)$. The $\mathrm{I}-\mathrm{E}$ and $\mathrm{I} / \mathrm{E}$ parameters of the deep breathing test were significantly lower in depression group compared to controls $(\mathrm{P}=0.021$, $\mathrm{P}=0.006$, respectively).

Table 1. Parameters of the heart rate variability and deep breathing test.

\begin{tabular}{cccc}
\hline Parameter & $\begin{array}{c}\text { Depression } \\
\text { group }\end{array}$ & $\begin{array}{c}\text { Control } \\
\text { group }\end{array}$ & Probability \\
\hline R-R interval (ms) & $800 \pm 34$ & $888 \pm 26$ & $\mathrm{P}=0.053$ \\
$\operatorname{logHF}-\mathrm{HRV}\left(\mathrm{ms}^{2}\right)$ & $6.16 \pm 0.29$ & $7.09 \pm 0.26$ & $\mathrm{P}=0.026$ \\
I-E (bpm) & $26.74 \pm 2.73$ & $34.93 \pm 1.81$ & $\mathrm{P}=0.021$ \\
I/E & $1.39 \pm 0.05$ & $1.59 \pm 0.03$ & $\mathrm{P}=0.006$ \\
\hline
\end{tabular}

Values are means \pm SE, logHF-HRV - logarithmic values of spectral powers in high (HF) bands, I - inspiration, E - expiration. 


\section{DisCUSSION}

Respiratory sinus arrhythmia (RSA) - the coupling of heart rate variability to the respiratory cycle - reflects the functional output of specific vagal pathways originating in the nucleus ambiguus that are neurophysiologically and neuroanatomically linked to several psychophysiological processes including emotion [4]. The heart rate variability spectral analysis allows to isolate the faster high frequency (HF) respiratory-coupled influences on the HRV $(0.15-0.4 \mathrm{~Hz})$ from slower sources of the HRV reflecting mainly sympathetic and parasympathetic activity via baroreflex. Therefore, a spectrally derived index of vagally mediated heart rate variability (HF-HRV) provides information about the respiratory sinus arrhythmia as a sensitive and non-invasive index of cardiac vagal function [1]. Additionally, since the respiratory mediated heart rate changes are small during quiet breathing, the deep breathing test is more convenient to evaluate RSA magnitude.

In adult depressive patients, a reduction in the HFHRV and lower parameters of the deep breathing test were found in comparison to healthy group [14]. Our results are in agreement with these findings and extend them into the adolescent group of MDD patients. Reduced respiratory sinus arrhythmia indicates cardiac vagal dysregulation in adolescent girls with major depressive disorder.

The mechanisms responsible for impaired modulation of cardiac autonomic activity and major depressive disorder are still discussed. Thayer and Lane $[6,7]$ provide the theoretical aspect of neurovisceral integration in emotion regulation/dysregulation emphasizing the neural correlates of vagal function and the role of brain in the regulation of the autonomic nervous system. One functional unit within the central nervous system (CNS) - central autonomic network [15] - includes the anterior cingulate, insular, and ventromedial prefrontal cortices, the central nucleus of amygdala, the paraventricular and related nuclei of the hypothalamus, the periaquaductal gray matter, the parabrachial nucleus, the nucleus of the solitary tract, the nucleus ambiguous, and specific medullary areas resulting in direct input to the sino-atrial node responsible for the complex variability that characterizes a healthy and adaptive cardiac time series [6]. We expect that these CNS areas partially overlap with the brain regions involved in the pressumed neuroanatomical basis of depression (e.g., prefrontal cortex or amygdala). Therefore, abnormalities in these centers involving other potential pathomechanisms (e.g., neurotransmitter dysregulation, hypothalamic-pituitary-adrenal axis dysfunction) might result in cardiac autonomic dysfunction $[16,17]$.

On the contrary, Rottenberg [18] revealed that the diagnosis of depression exerts a small-to-medium effect on cardiac vagal control, as indexed in lower RSA, and referred to confounding factors (e.g., sedentary behaviour) influencing cardiac autonomic function. We previously reported that RSA is reduced in obese adolescent subjects [19], and in the current study, we also evaluated a matched group of mentally healthy obese adolescent girls for comparison. We found that the magnitude of changes in the HF-HRV and I-E,
I/E parameters was similar between the adolescent patients with MDD and adolescent obese subjects. Thus, in agreement with Rottenberges study [18], we propose the effect of common factors associated with poor life-style (e.g., lack of physical activity) on cardiac vagal control in both disorders. It seems that the question of whether the reduced RSA indicating cardiac vagal dysfunction in the adolescent patients with MDD is a pure result of disrupted neurocardiac integrity or it is only a reflection of depression-related features and changed conditions (e.g., deficient social activity or reduced regular physical activities), remains unclear.

We conclude that adolescent girls with major depressive disorder have reduced respiratory sinus arrhythmia indicating impaired cardiac vagal regulation. The degree of impairment in these sick girls and in healthy obese subjects is similar. Since cardiac vagal dysregulation is associated with increased risk of cardiovascular morbidity, this finding underscores the importance of impaired autonomic neurocardiac integrity in adolescents with major depressive disorder without pharmacological treatment.

Acknowledgments: This study was supported by European Centre of Excellence Project Code 262201120016, Grants of Agency for Science and Ministry of Education (VEGA) No $1 / 2305 / 05$, No $1 / 4239 / 07$ and No $1 / 0064 / 08$. We thank M. Kollarik, Assoc. Prof., MD, PhD (Johns Hopkins University School of Medicine, Baltimore, USA) for the helpful comments on the manuscript.

Conflicts of interest: No conflicts of interest were declared by the authors in relation to this article.

\section{REFERENCES}

[1] Berntson GG, Bigger JT, Eckberg DL, Grossman P, Kaufmann PG, Malik M, Nagaraja HN, Porges SW, Saul JP, Stone PH, van der Molen MW (1997) Heart rate variability: Origins, methods, and interpretive caveats. Psychophysiology 34: 623-48. Review.

[2] Yasuma F, Hayano J (2004) Respiratory sinus arrhythmia: why does the heartbeat synchronize with respiratory rhythm? Chest 125: 683-90.

[3] Porges SW (1995) Orienting in a defensive world: Mammalian modification of our evolutionary heritage. A polyvagal theory. Psychophysiology 32: 301-18. Review.

[4] Porges SW (2007) The polyvagal perspective. Biol Psychol 74: 116-43. Review.

[5] Lane RD, Reiman EM, Ahern GL, Thayer JF (2001) Activity in medial prefrontal cortex correlates with vagal component of heart rate variability during emotion. Brain Cogn 47: 97-100. In: Thayer JF, Lane RD (2007) The role of vagal function in the risk for cardiovascular disease and mortality. Biol Psychol 74: 224-42. Review.

[6] Thayer JF, Lane RD (200) A model of neurovisceral integration in emotion regulation and dysregulation. J Affect Disord 61: 201-16.

[7] Thayer JF, Lane RD (20070 The role of vagal function in the risk for cardiovascular disease and mortality. Biol Psychol 74: 224-42. Review.

[8] Lehofer M, Moser M, Hoehn-Saric R, McLeod D, Liebmann P, Drnovsek B, Egner S, Hildebrandt G, Zapotoczky HG (1997) Major depression and cardiac autonomic control. Biol Psychiatry 42: 914-9.

[9] Nahshoni E, Aravot D, Aizenberg D, Sigler M, Zalsman G, Strasberg B, Imbar S, Adler E, Weizman A (2004) 
Heart rate variability in patients with major depression. Psychosomatics 45: 129-34.

[10] Yang TT, Simmons AN, Matthews SC, Tapert SF, Bischoff-Grethe A, Frank GKW, Arce E, Paulus MP (2007) Increased amygdala activation is related to heart rate during emotion processing in adolescent subjects. Neurosci Lett 428: 109-14.

[11] American Psychiatric Association. Diagnostic \& Statistical Manual of Mental Disorder DSM-IV-TR (Text Revision), 4th ed, American Psychiatric Association 2000.

[12] Task Force of the European Society of Cardiology and the North American Society of Pacing and Electrophysiology (1996) Heart rate variability. Standards of measurement, physiological interpretation, and clinical use. Circulation 93: 1043-65.

[13] Tarvainen MP, Ranta-Aho PO, Karjalainen PA (2002) An advanced detrending method with application to HRV analysis. IEEE Trans Biomed Eng 49: 172-5.

[14] Agelink MW, Boz C, Ullrich H, Andrich J (2002) Relationship between major depression and heart rate variability. Clinical consequences and implications for antidepressive treatment. Psychiatry Res 113: 139-49.

[15] Benarroch EE (1993) The central autonomic network: functional organization, dysfunction, and perspective. Mayo Clin Proc 68: 988-1001.
[16] Pajer KA (2007) Cardiovascular disease risk factors in adolescents: do negative emotions and hypothalamic-pituitary-adrenal axis function play a role? Curr Opin Pediatr 19: 559-64

[17] Brown ADH, Bartin DA, Lambert GW (2009) Cardiovascular abnormalities in patients with major depressive disorder. CNS Drugs 23: 583-602.

[18] Rottenberg J (2007) Cardiac vagal control in depression: A critical analysis. Biol Psychol 74: 200-11.

[19] Tonhajzerova I, Javorka M, Trunkvalterova Z, Chroma O, Javorkova J, Lazarova Z, Ciljakova M, Javorka K (2008) Cardio-respiratory interaction and autonomic dysfunction in obesity. J Physiol Pharmacol 59 Suppl 6: 70918.

\section{Corresponding author:}

Assoc. Prof. Ingrid Tonhajzerova

Institute of Physiology

Jessenius Faculty of Medicine

Comenius University

Mala Hora 4

03601 Martin

Slovakia

Phone/Fax: +421 434131426

E-mail: tonhajzerova@jfmed.uniba.sk 